Children's self-esteem was measured using Rosenberg's selfesteem scale (standardised). Arts engagement was measured through asking children how often they (1) listen to or play music, (2) draw, paint, or make things, and (3) read for enjoyment, outside school. Two sets of binary indicators were generated. Set A focused on whether children were engaged in these activities most days $(1=$ most days; $0=$ less often than most days). Set $B$ focused on extremes of engagement ( $1=$ most days; $0=$ never or less often than once a month). PSM models were implemented using kernel matching method; post-estimation test demonstrated high matching quality.

Results Results showed that all three activities were associated with higher levels of self-esteem when matching for all identified demographic, socio-economic and familial confounders (listen to/play music: $\mathrm{ATT}=0.07, \mathrm{SE}=0.02, \mathrm{p}<0.01$; paint, draw, and make things: ATT $=0.14, \mathrm{SE}=0.03, \mathrm{p}<0.001$; read for enjoyment: $\mathrm{ATT}=0.14, \mathrm{SE}=0.03, \mathrm{p}<0.001)$. The size of coefficients was doubled when the two comparison groups were more distinct with respect to reading frequency. Two sensitivity analyses additionally showed that (1) the relationship was more prominent when children engaged in these activities with their parents on a regular basis; and (2) there was no clear evidence that ability in either music or art activities moderated the relationship with self-esteem.

Conclusion Our findings show that arts activities have a significant association with children's self-esteem and that children may benefit more if their parents are also involved in the activities. We also find that the engagement itself offers a variety of benefits that enhance one's self-esteem, regardless of the ability in the activities. While PSM controls for observable factors, the causality of the association cannot be absolutely determined. However, the relevance of this research to the design and delivery of arts programmes for health is clear: arts engagement may well be important in supporting children's self-esteem - a core marker of positive life-long development.

\section{OP42 HOW MUCH EVIDENCE DO WE HAVE, AND HOW MUCH MORE DO WE NEED FOR ASSESSING THE IMPACT OF PUBLIC HEALTH INTERVENTIONS ON HEALTH INEQUALITIES? PART 1: SMOKING CESSATION INTERVENTIONS}

${ }^{1} \mathrm{~F}$ Yang ${ }^{*},{ }^{1} \mathrm{~A}$ Duarte, ${ }^{1} \mathrm{~S}$ Walker, ${ }^{2} \mathrm{C}$ Angus, ${ }^{2} \mathrm{D}$ Gillespie, ${ }^{1} \mathrm{~S}$ Griffin. ${ }^{1} \mathrm{C}$ entre for Health Economics, University of York, York, UKi ${ }^{2}$ Health Economics and Decision Science, ScHARR, University of Sheffield, Sheffield, UK

\subsection{6/jech-2019-SSMabstracts.61}

Background A distribution of public health intervention impact across socioeconomic groups can be estimated by examining the cumulative impact of socioeconomic differences across a staircase from need (e.g. prevalence) to intervention characteristics (e.g. effectiveness) using distributional cost effectiveness analysis (DCEA). The extent to which the amount of evidence for inequality at different steps of the staircase contributes to uncertainty in population level impact is not well understood. In this study, we used a DCEA of smoking cessation interventions to explore how socioeconomic inequality in model inputs impacts upon final conclusions about health inequality and value for money.

Methods A smoking cessation DCEA was developed to examine the impacts of interventions on different socioeconomic groups.

Impacts on total population health and health inequality were assessed using incremental population net health benefit (NHB) and incremental 'equally distributed equivalent' (EDE) health, both expressed in quality adjusted life years (QALYs). EDE reflects the extent by which the social value of NHB is reduced by inequality in its distribution that favours more advantaged groups.

Scenario analyses were used to explore: (i) the impact of ignoring socioeconomic differences in inputs, e.g., setting mortality in all groups to the average; (ii) the value of eliminating the differences by 'levelling up' uptake to the 'best'; and (iii) how the results differ when applying local level patterns of prevalence (e.g. York). The DCEA was adapted to reflect uncertainty in the extent of the differences between socioeconomic groups. Probabilistic sensitivity analysis (PSA) was used to determine the importance of uncertainty in each input for determining uncertainty in outputs.

Results Using English data, interventions improved NHB (Varenicline: 522,143 QALYs; e-cigarette: 334,874 QALYs) and EDE (Varenicline: 421,457 QALYs; e-cigarette: 270,097 QALYs), but increased health inequality (incremental $\mathrm{EDE}<$ incremental NHB). Setting mortality to the average, interventions provided an additional 4\% NHB and 2\% EDE. Setting uptake to the 'best', interventions provided an additional $33 \%$ NHB and 56-57\% EDE.

Using the data for York, there was uncertainty as to whether interventions reduced health inequality (probability: $16.9 \%$ for Varenicline and $22.6 \%$ for e-cigarette). The PSA indicated the key drivers for uncertainty were socioeconomic differences in effectiveness, smoking prevalence and uptake.

Conclusion Smoking cessation interventions provide value for money in all the scenarios and interventions to eliminate differences in uptake efficacy could provide additional EDE QALYs. Uncertainty in socioeconomic differences in smoking prevalence contributes the most to uncertainty about the health inequality impact of smoking cessation interventions.

\section{Health Inequalities 2}

\section{OP60 HOW MUCH EVIDENCE DO WE HAVE, AND HOW MUCH MORE DO WE NEED FOR ASSESSING THE IMPACT OF PUBLIC HEALTH INTERVENTIONS ON HEALTH INEQUALITIES? PART 2: ALCOHOL BRIEF INTERVENTIONS}

${ }^{1} \mathrm{C}$ Angus*, ${ }^{1} \mathrm{D}$ Gillespie, ${ }^{2} \mathrm{~F}$ Yang, ${ }^{2} \mathrm{~A}$ Duarte, ${ }^{2} \mathrm{~S}$ Walker, ${ }^{2} \mathrm{~S}$ Griffin. ${ }^{1}$ School of Health and Related Research, University of Sheffield, Sheffield, UK; ${ }^{2}$ Centre for Health Economics, University of York, York, UK

\subsection{6/jech-2019-SSMabstracts.62}

Background Inequalities in the impact of public health policies can be introduced at many stages of implementation, from need to effectiveness, and the net effect of any policy on health inequalities can be a combination of many, potentially smaller, inequalities. The extent to which these different 